\title{
Effect of Multimodal Pore Channels on Cargo Release from Mesoporous Silica Nanoparticles
}

\author{
Sushilkumar A. Jadhav, ${ }^{1,2}$ Valentina Brunella, ${ }^{1,2}$ Gloria Berlier, ${ }^{1,2}$ \\ Elena Ugazio, ${ }^{2,3}$ and Dominique Scalarone ${ }^{1,2}$ \\ ${ }^{1}$ Department of Chemistry, University of Torino, 10125 Torino, Italy \\ ${ }^{2}$ NIS Research Centre, University of Torino, 10125 Torino, Italy \\ ${ }^{3}$ Department of Pharmaceutical Science and Technology, University of Torino, 10125 Torino, Italy \\ Correspondence should be addressed to Sushilkumar A. Jadhav; sushil.unige@gmail.com \\ and Dominique Scalarone; dominique.scalarone@unito.it
}

Received 30 January 2016; Revised 11 May 2016; Accepted 12 May 2016

Academic Editor: Andrea Falqui

Copyright (C) 2016 Sushilkumar A. Jadhav et al. This is an open access article distributed under the Creative Commons Attribution License, which permits unrestricted use, distribution, and reproduction in any medium, provided the original work is properly cited.

\begin{abstract}
Mesoporous silica nanoparticles (MSNs) with multimodal pore channels were fully characterized by TEM, nitrogen adsorptiondesorption, and DLS analyses. MSNs with average diameter of $200 \mathrm{~nm}$ with dual pore channel zones with pore diameters of 1.3-2.6 and $4 \mathrm{~nm}$ were tested for their use in drug delivery application. Important role of the multimodal pore systems present on MSNs on the quantitative release of model drug ibuprofen was investigated. The results obtained revealed that the release profile for ibuprofen clearly shows distinct zones which can be attributed to the respective porous channel zones present on the particles. The fluctuations in the concentration of ibuprofen during the prolonged release from MSNs were caused by the multimodal pore channel systems.
\end{abstract}

\section{Introduction}

Mesoporous silica nanoparticles (MSNs) represent one of the most versatile reservoir materials used for delivery of different molecules. The versatility comes from stability, biocompatibility, highly ordered porosity, and large surface area of this material [1]. In particular MSNs have shown great promise in their use for drug delivery [2-4], targeted drug delivery [5], improved solubility and delivery of poorly soluble drugs [6], $\mathrm{pH}$ responsive drug delivery systems [7], and controlled release of drugs [8]. In all these applications MSNs can be used as reservoir for drug molecules, and prolonged and controlled release of the drug in different media can be obtained by diffusion. Several molecules from different classes of drugs such as anti-inflammatory, anticancer drugs, and antioxidants were loaded inside MSNs and their release kinetics was studied $[9,10]$. The extended studies have revealed that there are several factors which influence drug loading, stability of the material, and drug release. Altogether these factors determine the efficiency of MSNs in the delivery of the desired drugs in different environments. The most commonly investigated factors which greatly influence the delivery of molecules are size of the particles and porosity $[11,12]$, pore length, pore volume and pore diameter [13], morphology [14], pore size [15], and loading capacity and their hydrothermal stability [16]. Improved control over the release of the drugs which allows tuning of the release profiles can be achieved by trigger mechanisms activated by various organic functional groups or smart polymers grafted on the porous particles [17]. However fewer investigations are reported which go into the details of the orientations of the microstructures which are multimodal pore systems that may get formed during the synthesis of MSNs. In last few years several optimized hydrothermal or sol-gel synthesis methods for the synthesis of MSNs, which yield monodisperse particles with improved control over the obtained porosity, pore length or pore volume, and orientations of the mesochannels, have been reported [18, 19]. Various factors such as the use of core swelling (pore extending) agents together with the traditional micelle forming agents, the choice of the alkoxysilane itself, which undergoes hydrolysiscondensation reaction to give rise to the siloxane network, 
and rate of the hydrolysis of alkoxysilane are investigated to study their influence on size of the nanoparticles and orientation of the mesochannels [20]. High resolution images of the MSNs obtained from electronic microscope reveal that there may exist arrays of mesochannels forming distinct zones inside the same particle. Such complicated multimodal pore orientations may result from use of different ratios of starting materials, concentrations of the by-product formed, and synthesis conditions used.

Most of the problems associated with the synthesis and the choice of parameters which can lead to the monodisperse MSNs with desired structural features and without any defects or additional pore mesochannels can be resolved in small or laboratory scale synthesis. However large or commercial scale synthesis of MSNs faces a big challenge of producing reasonably monodisperse particles within a specific range of diameter without any major structural modifications that may arise during their scale-up [21]. Most of the reports about mesoporous silica synthesis use cationic surfactants such as cetyltrimethylammonium bromide (CTAB) in basic medium as a soft template for obtaining most popular MCM41 type particles with 2D hexagonal mesostructures which have pore diameters around $3 \mathrm{~nm}$. For increasing the pore diameter and further tuning the porous structure, a dual surfactant system is used which contains a cationic surfactant together with an amphiphilic triblock copolymer or micelle core swelling molecules, such as trimethylbenzenes (TMBs), with CTAB. The presence of two templates actually can lead to complications like the formation of multioriented mesochannels which can also give core-shell structured MSNs with dually oriented mesochannels [22]. In addition to the cotemplate another molecule which can lead to the formation of dual mesochannels in MSNs is alcohol (mostly ethanol) which is either used as a cosolvent in the synthesis or formed during the hydrolysis of commonly used silica source (e.g., tetraethylorthosilicate). Structural modifications caused during the synthesis and formation of dually oriented porous mesochannels can have considerable effect on loading and more importantly on the release of the molecules loaded inside such MSNs. In drug delivery applications normally the loading of drug molecules inside MSNs is carried out by soaking them in the concentrated solution of the drug molecule at specific temperature until almost all the mesopores of the MSNs are filled. Each mesochannel present on the particle represents a distinct zone which can show different release kinetics for the molecule loaded inside it. The release kinetics will depend upon size of the particle, size of this mesochannel zone with respect to the overall size of the particles, and diameter of the pores in this zone. These variations in the release kinetics play an important role in real application of MSNs in drug delivery as they can greatly alter the concentration of a drug released at different time intervals. Sometimes the change in concentration of released drug is directly linked to other factors, such as solubility of the drug molecule in the release medium (water, buffer, saline solution, etc.), and to the efficiency of the trigger mechanism. Instead the change in concentration of the drug released by MSNs may be due to the presence of secondary microstructures on them.
The objectives of the study were to fully characterize recently marketed MSNs with multimodal pore channels to get important information about their structure and properties which are not reported in detail neither by the producers nor in the literature and to test their suitability for drug delivery application. On the basis of the results obtained, it was confirmed that a careful examination of synthesized or commercially available MSNs is needed for their use in drug delivery applications.

\section{Experimental}

2.1. Materials, Methods, and Instruments. MSNs (particle size $200 \mathrm{~nm}$, pore size $4 \mathrm{~nm}$ ), ibuprofen, and hexane were purchased from Sigma-Aldrich, Italy. Ibuprofen and hexane were used as received and MSNs were degassed before analysis.

High Resolution Transmission Electron Microscopy (HRTEM) images were obtained with a JEOL 2010 instrument $(300 \mathrm{kV})$ equipped with a LaB6 filament. For specimen preparation powdery samples were supported onto holed carbon coated copper grids by dry deposition.

Fourier Transform Infrared Spectroscopy (FTIR) spectra were collected with a Perkin Elmer FTIR Nexus instrument equipped with an attenuated total reflectance (ATR) devise (Thermo Nicolet Smart Endurance) and with a DTGS detector. Spectra were collected in the spectral range of 4000$670 \mathrm{~cm}^{-1}$ and with a resolution of $4 \mathrm{~cm}^{-1}$.

Gas-volumetric analysis, specific surface area (SSA), and pore volume and size were measured by $\mathrm{N}_{2}$ adsorption-desorption isotherms at $77 \mathrm{~K}$ using an ASAP 2020 (Micromeritics) gas-volumetric analyzer. SSA was calculated using the Brunauer-Emmett-Teller (BET) method. Porosity distribution, allowing estimating width and volume, was calculated with the Nonlocal Density Functional Theory (NLDFT), employing the $\mathrm{N}_{2}$-DTF model with slit pores geometry (regularization low). Prior to analyses, samples were kept in oven at $50^{\circ} \mathrm{C}$ for 4 hours and then outgassed at RT overnight. Thermogravimetric analyses (TGA) were carried out on a TA Q500 model from TA Instruments by heating samples contained in alumina pans at a rate of $10^{\circ} \mathrm{C} / \mathrm{min}$ from 25 to $800^{\circ} \mathrm{C}$ in air.

Dynamic light scattering (DLS) measurements were carried out by using a Malvern ZS 90 Zetasizer instrument. $0.1 \%$ suspensions of nanoparticles were prepared in deionized water and sonicated for 20 minutes before the analysis. The measurements of hydrodynamic diameter (HD) and zeta potential $(\mathrm{Zp})$ were performed in triplicate for each sample and the mean value is reported. The measurements were carried out at $20^{\circ} \mathrm{C}$. Elemental analysis was performed with Flash EA 1112 series (CHNS analyzer) instrument from Thermo Electron Co. with vanadium pentaoxide as catalyst.

UV-Visible analyses were performed using a Lambda 15 UV-Vis spectrophotometer (Perkin Elmer). For ibuprofen quantification a calibration curve $\left(\varepsilon=185.1 \mathrm{M}^{-1}, R^{2}=0.99\right)$ was obtained by plotting absorbance versus concentration of ibuprofen between $6.0 \times 10^{-5}$ and $4.8 \times 10^{-4} \mathrm{M}$.

2.2. Drug Loading and Release Tests. MSNs were outgassed at $\mathrm{RT}$ overnight to remove adsorbed water and then dispersed 


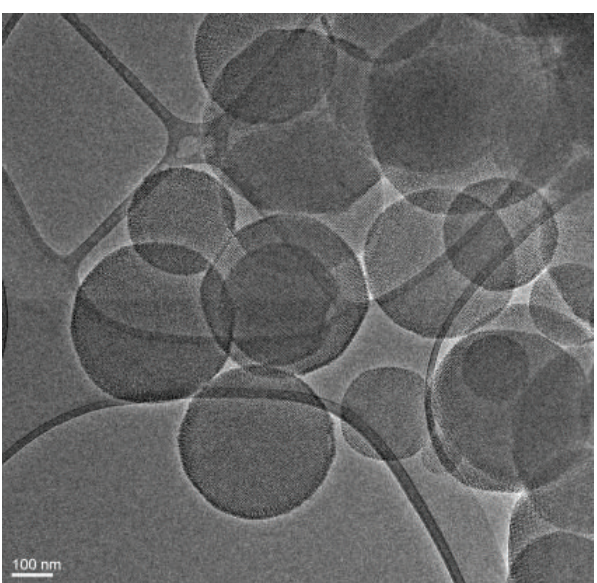

(a)

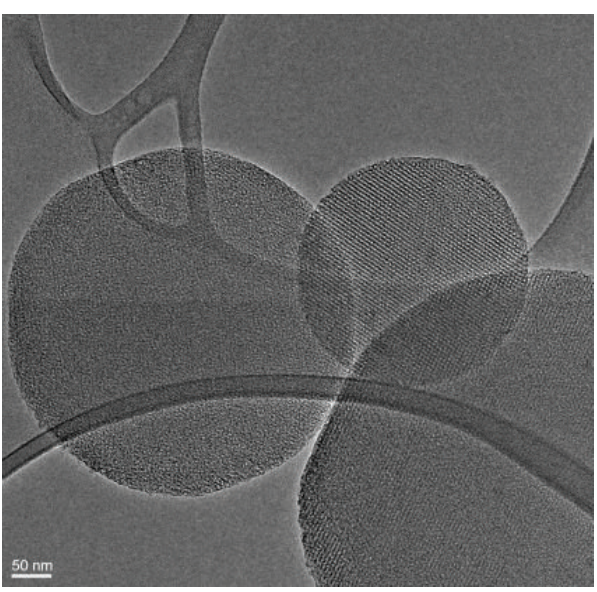

(c)

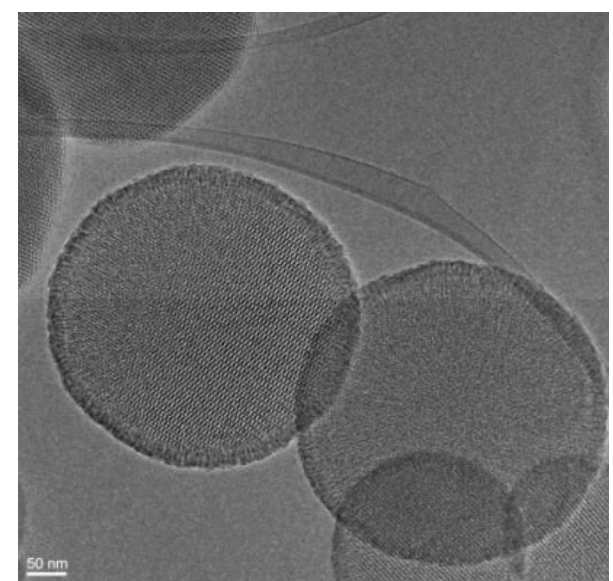

(b)

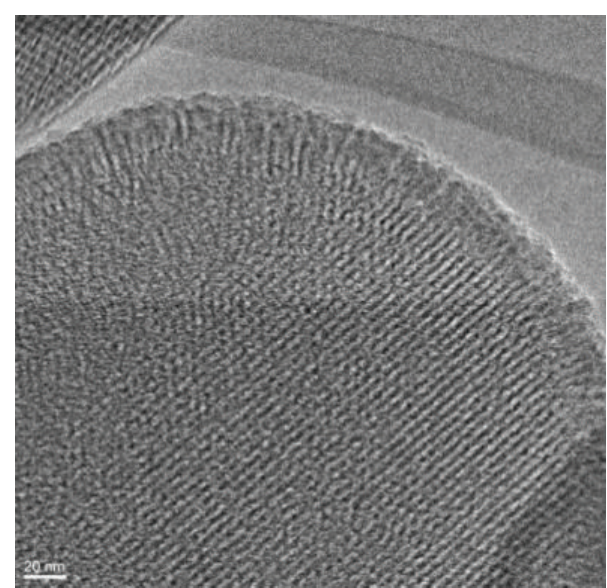

(d)

FIGURE 1: TEM images at different magnification showing the porous structure of the MSNs and the different orientation of the pore channels at the periphery and in the inner part of the MSNs.

under magnetic stirring in a hexane solution $(0.16 \mathrm{M})$ of ibuprofen at $40^{\circ} \mathrm{C}$ for 48 hours. Then nanoparticles were separated by centrifugation, washed with hexane, and dried at RT. Ibuprofen loading was determined by TGA and UV-Vis spectroscopy by monitoring the absorbance at $272 \mathrm{~nm}$. The loading was calculated from TGA.

In vitro drug release tests were carried out in $0.9 \% \mathrm{NaCl}$ solution in water in a constant temperature box equipped with multiposition magnetic stirrer. The release profiles in saline solution were obtained by monitoring the absorbance of ibuprofen at $272 \mathrm{~nm}$ at different time intervals and temperatures. Each time the supernatant solution of ibuprofen was separated from the particles by centrifugation of the suspension.

\section{Results and Discussion}

The HR-TEM images of the MCM-41 type MSNs which are used in this study are shown in Figures $1(\mathrm{a})-1(\mathrm{~d})$. The TEM images revealed that the mesoporous silica nanoparticles are with diameters ranging from 60 to $200 \mathrm{~nm}$. The ordered porous structure is clearly visible with pore channels running throughout the body of the particles. Magnified
TEM image of a single particle is shown in Figure 1(d). A border of approximately $15-25 \mathrm{~nm}$ around each particle is observed which clearly shows that the orientation of the pore channels is different at the periphery with respect to the central mesochannels indicating a dual mesochannel type of structure.

In order to further confirm the dual porous structure of the particles, nitrogen adsorption-desorption analyses on the MSNs samples were carried out. The adsorption-desorption isotherms (Figure 2) showed a complex profile with both features of type I and type IV isotherms. Namely, the steep knee at low pressure $\left(<0.01-0.02 p / p^{0}\right)$ is typical of microporous materials, while the capillary condensation step and related hysteresis loop in the $0.4-0.5$ pressure range indicated the presence of mesopores [23]. This is reflected in the pore diameter distribution curve, calculated by NLDFT, which clearly showed the presence of micropores and mesopores in the particles, and it also indicates the predominant presence of mesopores of $4 \mathrm{~nm}$ in diameter. Specific surface area of the particles calculated by BET method was $901 \mathrm{~m}^{2} / \mathrm{g}$.

Dynamic light scattering (DLS) analyses were performed on very dilute suspensions of MSNs. The particle size 




(a)

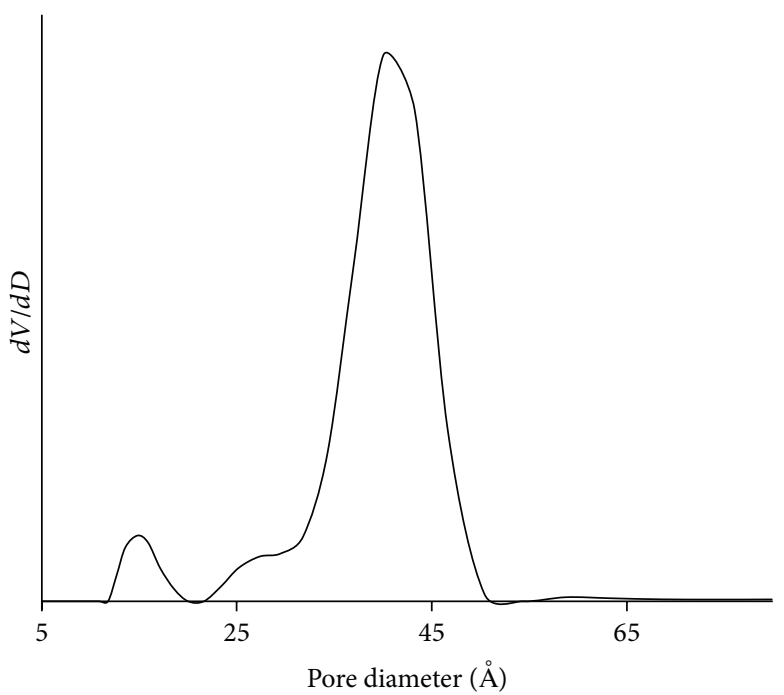

(b)

FIGURE 2: $\mathrm{N}_{2}$ adsorption- (squares) desorption (triangles) isotherms (a) and the corresponding pore size distribution curve of MSNs (b).

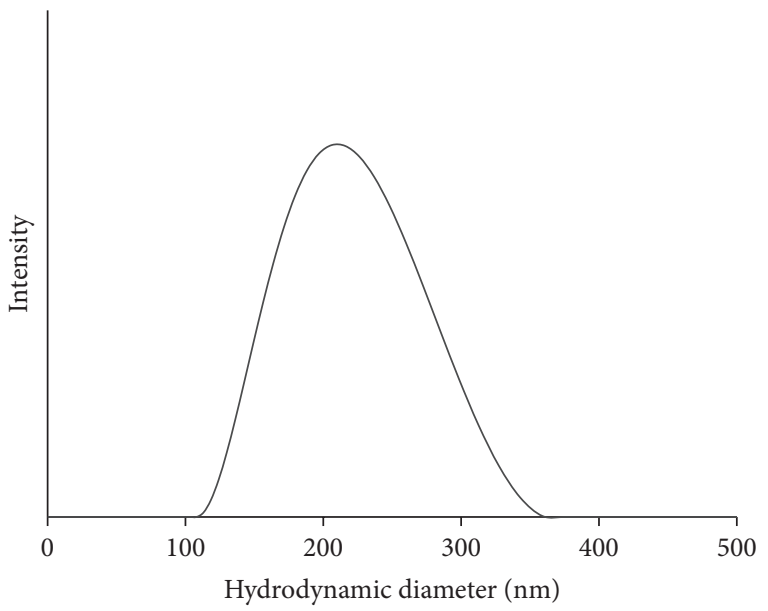

FIGURE 3: Particle size distribution of MSNs by DLS analysis.

distribution curve is shown in Figure 3. The average size of the particles was in the range of $206-220 \mathrm{~nm}$. The particles showed high aggregation tendency and bigger aggregates were immediately formed. The polydispersity index (PDI) of all the samples subjected to DLS analysis was 0.3 which with the time due to fast aggregation of bare particles increased to almost 1.0 confirming high aggregation rate of the bare MSNs. The zeta potential value at $\mathrm{pH}$ around $7.2 \mathrm{was}-17.3 \mathrm{mV}$, which is actually lower than the values reported for MSNs [24-27]. The number of silanol groups determines the reactivity of the material (silica) and is reflected in the zeta potential value obtained by DLS analysis. The surface silanols also play an important role in drug loading as a higher number of silanol groups can hold more drug through secondary interactions with the polarizable groups present on the drug molecules [28]. The elemental analysis of particles showed only the

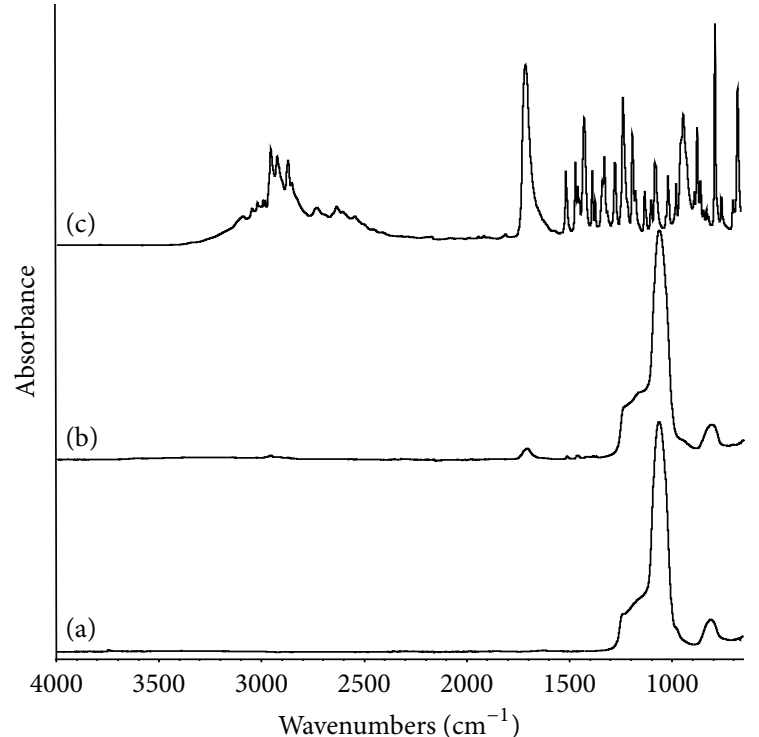

FIGURE 4: ATR-FTIR spectra of (a) MSNs, (b) ibuprofen loaded MSNs, and (c) ibuprofen.

presence of trace amount of carbon $(0.40 \%)$ and hydrogen $(0.48 \%)$ confirming chemical purity of the particles.

Loading of model anti-inflammatory drug ibuprofen was carried out by soaking MSNs in concentrated $(0.16 \mathrm{M})$ solution of ibuprofen in hexane with constant stirring of the solution with suspended particles for 48 hours at $40^{\circ} \mathrm{C}$. After loading, the presence of ibuprofen inside the mesopores of silica was analyzed by ATR-FTIR spectroscopy and the results are shown in Figure 4. The ibuprofen loaded MSNs showed the presence of peaks of the drug; in particular the peak due to $\mathrm{C}=\mathrm{O}$ absorption at $1730 \mathrm{~cm}^{-1}$ was clearly evident. The quantitative loading of ibuprofen was determined by TGA and was $\sim 25.25 \%$ (Figure 5 ) value obtained after 


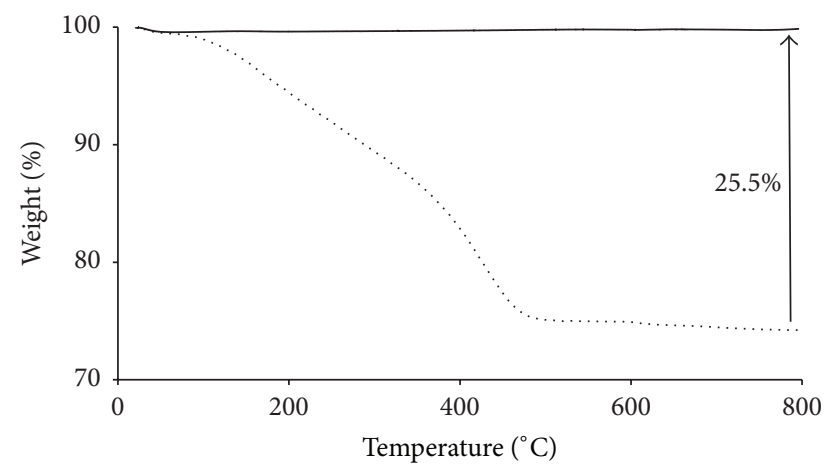

FIGURE 5: TGA curves of MSNs (solid line) and MSNs-ibuprofen loaded (dotted line).

subtracting the weight loss due to silica. The weight loss upon programmed heating up to $800^{\circ} \mathrm{C}$ for MSNs was only $0.25 \%$, while the weight loss observed for the ibuprofen loaded particles was $25.5 \%$. The increased weight loss is due to the loaded ibuprofen. In vitro drug release tests were performed in saline solution $(0.9 \% \mathrm{NaCl}$ solution in $\mathrm{DI}$ water) by using the different aliquot method with varying amount of the saline solution (i.e., the amount is adjusted in order to avoid saturation of the solution with ibuprofen at prolonged release time). The quantitative release of ibuprofen from the particles was determined by UV-Vis spectroscopy. The ibuprofen release profiles obtained at two different temperatures are shown in Figures 6(a) and 6(b).

The release tests were also performed at low temperature just to exclude the possibility of change in concentration of the released drug due to minor temperature changes during the magnetic stirring of the suspensions in saline solution. The quantitative ibuprofen release curves showed three distinct zones, that is, (A), (B), and (C), which can be correlated with different parts of the MSNs that are outer surface (A), the thin microporous layer at the periphery of particle (B), and the inner mesoporous part of particle (C) (Figure 6(c)). Accordingly, release curves are characterized by the following three stages: (A) the first stage which involves the rapid release of ibuprofen within the first 45-60 min, owing to the release of ibuprofen adsorbed on the external surface of the particles. Physisorption of ibuprofen molecules is possible in almost all the methods (impregnation or loading from melt) used for loading $[29,30]$. Although it is expected that most of the ibuprofen molecules should be loaded inside the mesopores, certain amount of ibuprofen is always found to remain physisorbed on the surface of the particles. Even repeated washing with the solvent used for loading sometimes cannot remove completely the physisorbed drug because of the strong interactions of the carboxylic group present on the molecule with the surface silanol (Si-OH) groups. To break this interaction water is needed as it is well known that water forms a bilayer on silica surface that interrupts the ibuprofen silica surface interactions. As organic solvents such as hexane or ethanol are used for the loading of ibuprofen the possibility of breaking interactions is not there and thus ibuprofen remains strongly physisorbed on the surface of the particles. However for the release experiments, buffer systems or water is used as a solvent and hence the first delivery of cargo is the amount of ibuprofen physisorbed on the particles. (B) After the first step, the concentration of drug decreases slightly and increases slowly again from 1 to 5 hours. It is reasonable to attribute this release to those ibuprofen molecules which are trapped/loaded in the thin shell at the periphery of the particles where (micro)pores have smaller diameter and different orientation compared to inner mesopores (Figure 6(c)). Although the thickness of this zone is small, because of the small diameter of the pores, a prolonged release of the drug is observed for approximately 4 hours. This release time can be tuned by tuning the diameter of these micropores. (C) Finally, the ibuprofen concentration increases again and attains a constant value after 25 hours (see particularly the release curve obtained at $40^{\circ} \mathrm{C}$, shown in Figure 6(a)). The release in the last stage is due to the drug molecules loaded in the mesopores which are long in length. Such observation of fluctuations in the concentration of the loaded drug molecules is common for certain porous materials, but the interpretation of the change in concentration of the drugs is mostly related to the physisorption of the drug without many details of influence of the microstructures. With the nonconventional release profiles obtained for MSNs used in this study it is quite evident that the multimodal pore system plays an important role in the drug release behaviour. Quantities of ibuprofen released after each phase (A) (after $45 \mathrm{~min}$ ), (B) (after $5 \mathrm{~h}$ ), and (C) (after $48 \mathrm{~h}$ ) are shown in Figure 7.

Although this explanation about the change in the concentration of the drug released with time appears as a possible shortcoming (or defect) of the material under study, it on the other hand can also prove to be crucial where such fluctuations in the concentration of the drug are desired with time. Multimodal mesochannel systems can thus be generated on MSNs to tune the dose of the drugs released with time. For this reason some multimodal pore systems in some studies are created purposely on the porous particles. Niu et al. [23] showed that with careful modification of the synthesis condition and with choice of templates used it is possible to obtain multimodal porous particles with desired pore sizes and pore length. Manipulation of synthesis conditions and concentrations of templates can be done to tune the properties of the pores, which is recently termed as the "pore engineering" [22].

\section{Conclusion}

MSNs used in this study were fully characterized and tested for their use in drug delivery applications. The results on characterization of MSNs provide important additional information about size and porous structure of the material which is important for their use in different applications. From the results on drug release it is proved that multimodal pore systems on mesoporous silica can influence the quantitative release (concentration) of the drug loaded. This release depends upon the size of the mesochannel zone with respect to the overall size of the particle and diameter of the pores in different mesochannels. After initial fluctuations in 

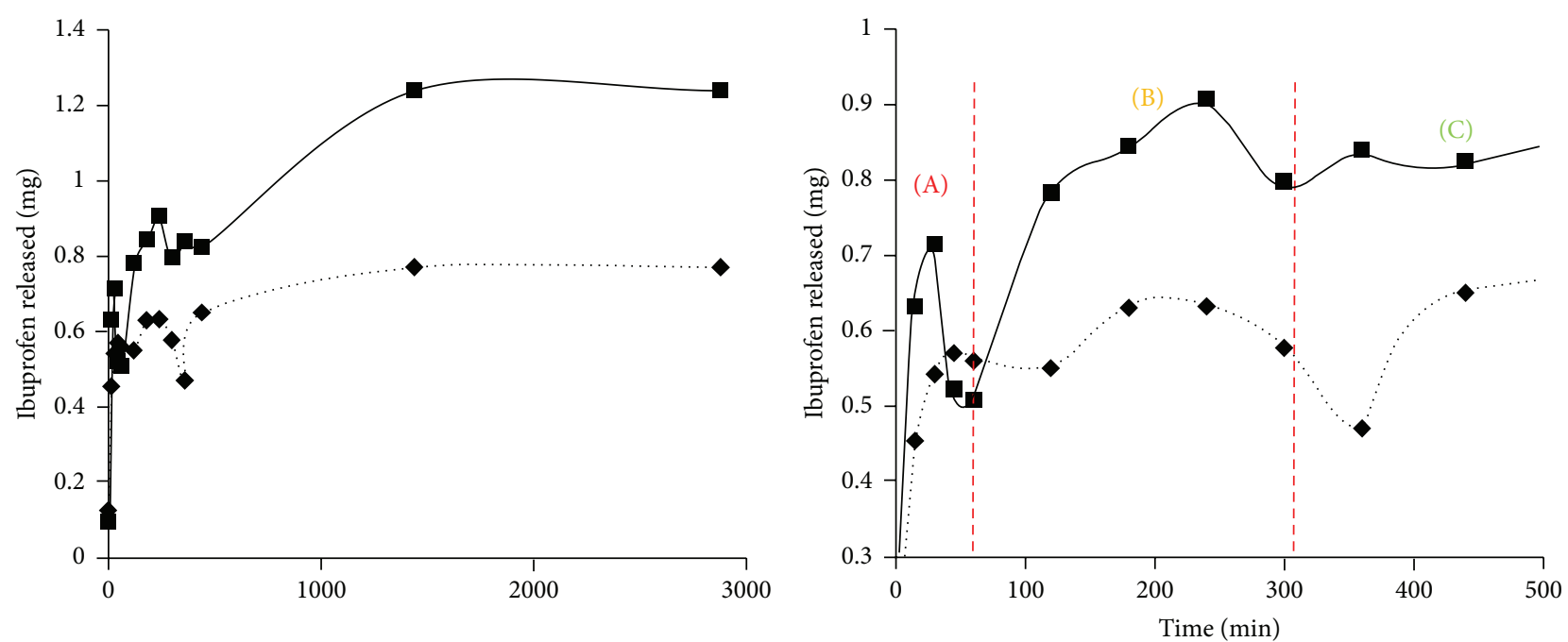

(a)

(b)



(c)

Figure 6: (a) Ibuprofen release curves at $4^{\circ} \mathrm{C}$ (dotted line with diamonds) and $40^{\circ} \mathrm{C}$ (solid line with squares); (b) release curves up to 500 minutes. Areas labelled as (A), (B), and (C) correlated with the different parts of the MSN as shown in (c).

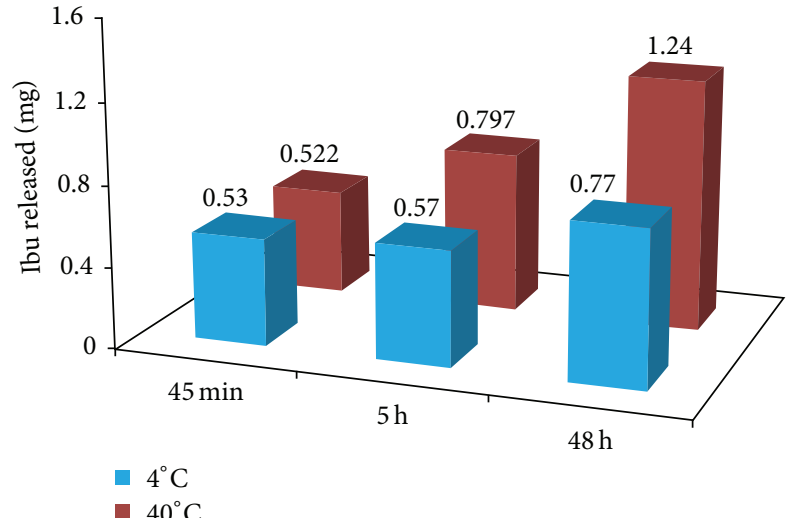

FIgURE 7: Quantity of ibuprofen released at three time intervals corresponding to zones (A) (45 min), (B) (5 h), and (C) $(48 \mathrm{~h})$ of the release profiles as showed in Figure 6.

the concentration of the drug a prolonged release of the active molecule depending on the size of mesochannel zone and core pore length of the particles was obtained. This study points out that the presence of or formation of "unwanted" microstructures on mesoporous materials during the synthesis is an important factor to take into consideration before their use for drug delivery applications.

\section{Competing Interests}

The authors declare that there are no competing interests regarding the publication of this paper.

\section{Acknowledgments}

Compagnia di San Paolo and University of Torino, Italy, are gratefully acknowledged for funding Project ORTO114XNH through "Bando per il Finanziamento di Progetti di Ricerca di Ateneo, Anno 2011." The authors would like to thank Dr. F. Caldera for elemental analyses. Dr. Sushilkumar A. Jadhav acknowledge MIUR, Italy, for financial support. 


\section{References}

[1] S. A. Jadhav, "Incredible pace of research on mesoporous silica nanoparticles," Inorganic Chemistry Frontiers, vol. 1, no. 10, pp. 735-739, 2014.

[2] Y. Wang, Q. Zhao, N. Han et al., "Mesoporous silica nanoparticles in drug delivery and biomedical applications," Nanomedicine: Nanotechnology, Biology, and Medicine, vol. 11, no. 2, pp. 313-327, 2015.

[3] C. Argyo, V. Weiss, C. Bräuchle, and T. Bein, "Multifunctional mesoporous silica nanoparticles as a universal platform for drug delivery," Chemistry of Materials, vol. 26, no. 1, pp. 435-451, 2014.

[4] M. Colilla and M. Vallet-Regí, "Responsive mesoporous silica nanoparticles for targeted drug delivery," RSC Smart Materials, vol. 14, no. 1, pp. 136-166, 2015.

[5] A. Baeza, M. Colilla, and M. Vallet-Regí, "Advances in mesoporous silica nanoparticles for targeted stimuli-responsive drug delivery," Expert Opinion on Drug Delivery, vol. 12, no. 2, pp. 319-337, 2015.

[6] J. Li, L. Xu, B. Yang et al., "Facile synthesis of functionalized ionic surfactant templated mesoporous silica for incorporation of poorly water-soluble drug," International Journal of Pharmaceutics, vol. 492, no. 1-2, pp. 191-198, 2015.

[7] Y. Tian, Y. Kong, X. Li, J. Wu, A. C.-T. Ko, and M. Xing, "Light- and $\mathrm{pH}$-activated intracellular drug release from polymeric mesoporous silica nanoparticles," Colloids and Surfaces B: Biointerfaces, vol. 134, pp. 147-155, 2015.

[8] I. I. Slowing, J. L. Vivero-Escoto, C.-W. Wu, and V. S.-Y. Lin, "Mesoporous silica nanoparticles as controlled release drug delivery and gene transfection carriers," Advanced Drug Delivery Reviews, vol. 60, no. 11, pp. 1278-1288, 2008.

[9] S. K. Natarajan and S. Selvaraj, "Mesoporous silica nanoparticles: importance of surface modifications and its role in drug delivery," RSC Advances, vol. 4, no. 28, pp. 14328-14334, 2014.

[10] N. Vadia and S. Rajput, "Mesoporous material, MCM-41: a new drug carrier," Asian Journal of Pharmaceutical and Clinical Research, vol. 4, no. 2, pp. 44-53, 2011.

[11] P. Burguete, A. Beltrán, C. Guillem et al., "Pore length effect on drug uptake and delivery by mesoporous silicas," ChemPlusChem, vol. 77, no. 9, pp. 817-831, 2012.

[12] G. Gonzalez, A. Sagarzazu, and T. Zoltan, "Infuence of microstructure in drug release behavior of silica nanocapsules," Journal of Drug Delivery, vol. 2013, Article ID 803585, 8 pages, 2013.

[13] V. Cauda, L. Mühlstein, B. Onida, and T. Bein, “Tuning drug uptake and release rates through different morphologies and pore diameters of confined mesoporous silica," Microporous and Mesoporous Materials, vol. 118, no. 1-3, pp. 435-442, 2009.

[14] F. Qu, G. Zhu, H. Lin et al., "A controlled release of ibuprofen by systematically tailoring the morphology of mesoporous silica materials," Journal of Solid State Chemistry, vol. 179, no. 7, pp. 2027-2035, 2006.

[15] P. Horcajada, A. Rámila, J. Pérez-Pariente, and M. Vallet-Regí, "Influence of pore size of MCM-41 matrices on drug delivery rate," Microporous and Mesoporous Materials, vol. 68, no. 1-3, pp. 105-109, 2004.

[16] J. Andersson, J. Rosenholm, S. Areva, and M. Lindén, "Influences of material characteristics on ibuprofen drug loading and release profiles from ordered micro- and mesoporous Silica matrices," Chemistry of Materials, vol. 16, no. 21, pp. 4160-4167, 2004.
[17] P. Yang, S. Gai, and J. Lin, "Functionalized mesoporous silica materials for controlled drug delivery," Chemical Society Reviews, vol. 41, no. 9, pp. 3679-3698, 2012.

[18] H. Yamada, C. Urata, E. Yamamoto, S. Higashitamori, Y. Yamauchi, and K. Kuroda, "Effective use of alkoxysilanes with different hydrolysis rates for particle size control of aqueous colloidal mesostructured and mesoporous silica nanoparticles by the seed-growth method," ChemNanoMat, vol. 1, no. 3, pp. 194-202, 2015.

[19] F. Tang, L. Li, and D. Chen, "Mesoporous silica nanoparticles: synthesis, biocompatibility and drug delivery," Advanced Materials, vol. 24, no. 12, pp. 1504-1534, 2012.

[20] S.-H. Wu, C.-Y. Mou, and H.-P. Lin, "Synthesis of mesoporous silica nanoparticles," Chemical Society Reviews, vol. 42, no. 9, pp. 3862-3875, 2013.

[21] K. Zang, L. L. Xu, J. G. Jiang et al., "Facile large-scale synthesis of monodisperse mesoporous silica nanospheres with tunable pore structure," Journal of American Chemical Society, vol. 135, no. 7, pp. 2427-2430, 2013.

[22] J. Peng, J. Liu, J. Liu, Y. Yang, C. Li, and Q. Yang, "Fabrication of core-shell structured mesoporous silica nanospheres with dually oriented mesochannels through pore engineering," Journal of Materials Chemistry A, vol. 2, no. 21, pp. 8118-8125, 2014.

[23] D. Niu, Z. Ma, Y. Li, and J. Shi, "Synthesis of core-shell structured dual-mesoporous silica spheres with tunable pore size and controllable shell thickness," Journal of the American Chemical Society, vol. 132, no. 43, pp. 15144-15147, 2010.

[24] P. DeMuth, M. Hurley, C. Wu, S. Galanie, M. R. Zachariah, and P. DeShong, "Mesoscale porous silica as drug delivery vehicles: synthesis, characterization, and $\mathrm{pH}$-sensitive release profiles," Microporous and Mesoporous Materials, vol. 141, no. 1-3, pp. 128-134, 2011.

[25] L. Yuan, Q. Tang, D. Yang, J. Z. Zhang, F. Zhang, and J. Hu, "Preparation of $\mathrm{pH}$-responsive mesoporous silica nanoparticles and their application in controlled drug delivery," Journal of Physical Chemistry C, vol. 115, no. 20, pp. 9926-9932, 2011.

[26] G.-F. Luo, W.-H. Chen, Y. Liu, Q. Lei, R.-X. Zhuo, and X.-Z. Zhang, "Multifunctional enveloped mesoporous silica nanoparticles for subcellular co-delivery of drug and therapeutic peptide," Scientific Reports, vol. 4, article 6064, pp. 1-10, 2014.

[27] W. Xu, J. Riikonen, T. Nissinen et al., "Amine surface modifications and fluorescent labeling of thermally stabilized mesoporous silicon nanoparticles," Journal of Physical Chemistry C, vol. 116, no. 42, pp. 22307-22314, 2012.

[28] N. H. N. Kamarudin, A. A. Jalil, S. Triwahyono et al., "Elucidation of acid strength effect on ibuprofen adsorption and release by aluminated mesoporous silica nanoparticles," RSC Advances, vol. 5, no. 38, pp. 30023-30031, 2015.

[29] C. Charnay, S. Bégu, C. Tourné-Péteilh, L. Nicole, D. A. Lerner, and J. M. Devoisselle, "Inclusion of ibuprofen in mesoporous templated silica: drug loading and release property," European Journal of Pharmaceutics and Biopharmaceutics, vol. 57, no. 3, pp. 533-540, 2004.

[30] E. Skorupska, A. Jeziorna, P. Paluch, and M. J. Potrzebowski, "Ibuprofen in mesopores of mobil crystalline material 41 (MCM-41): a deeper understanding," Molecular Pharmaceutics, vol. 11, no. 5, pp. 1512-1519, 2014. 

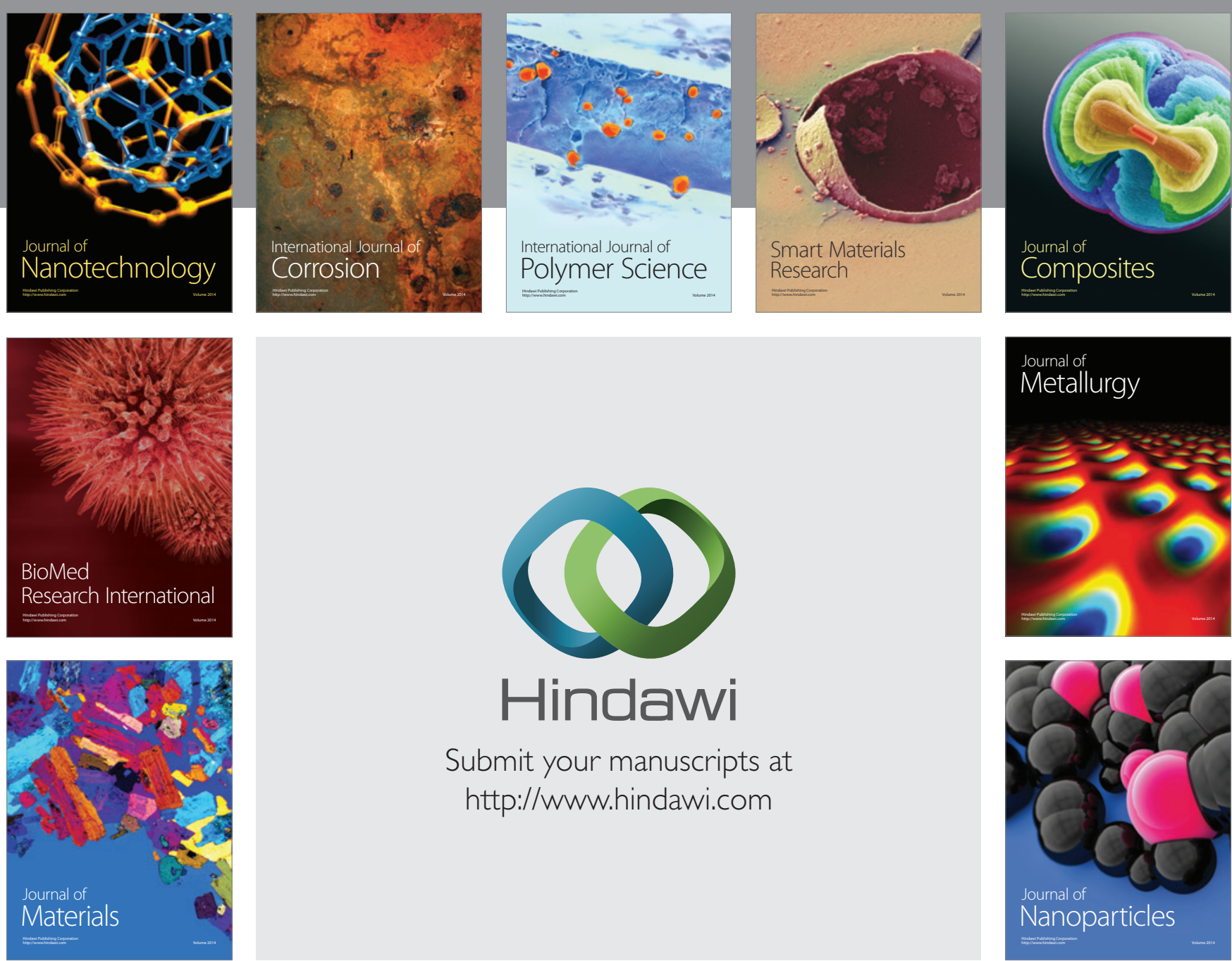

\section{Hindawi}

Submit your manuscripts at

http://www.hindawi.com

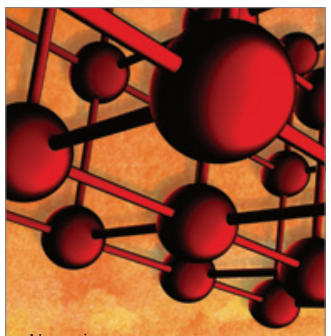

Materials Science and Engineering
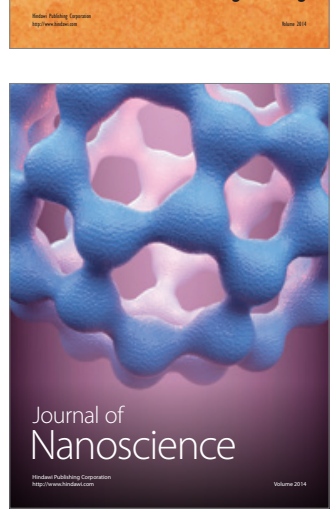
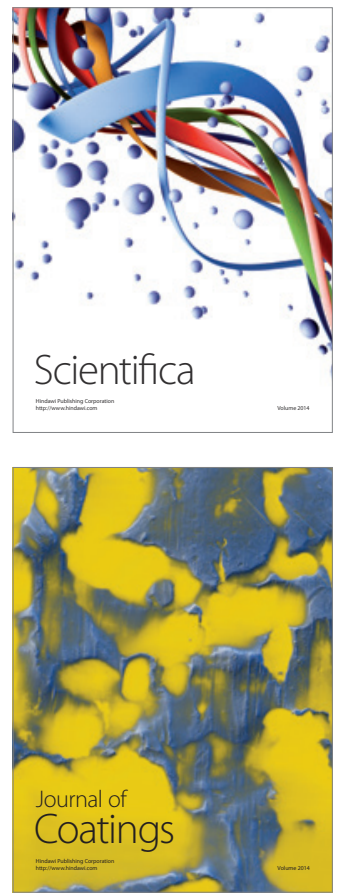
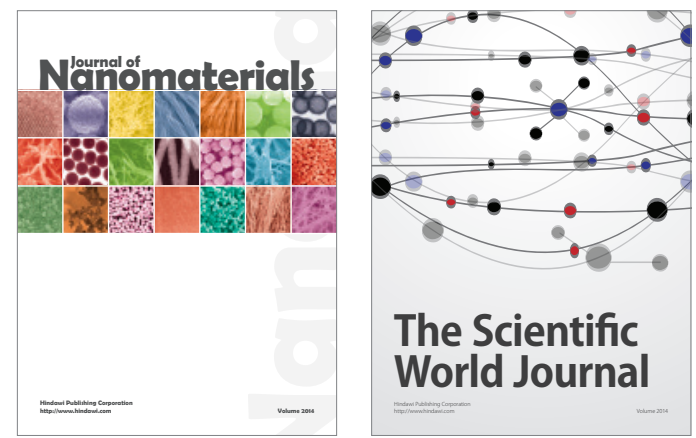

The Scientific World Journal
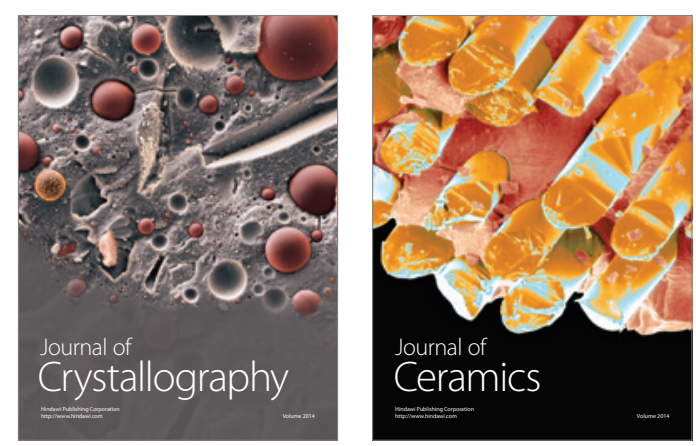
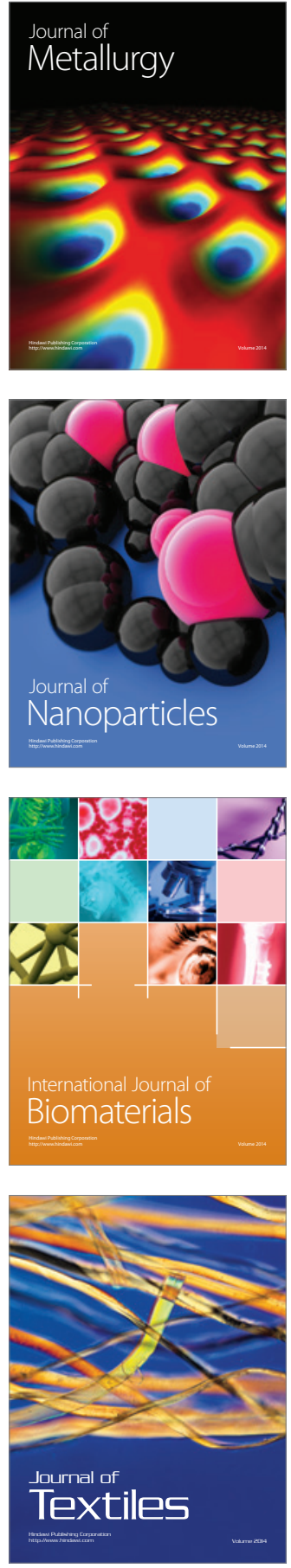